\title{
A Paraneoplastic Syndrome to Remember: A Case of Disseminated Intravascular Coagulation in Lung Cancer
}

\author{
$\underline{\text { Rita Gameiro }}^{1}$, Rodolfo Gomes ${ }^{1}$, Vanda Jorge ${ }^{2}$, Bárbara Picado ${ }^{1}$, Fernando Martos Gonçalves ${ }^{1}$, José Lomelino Araújo ${ }^{1}$ \\ ${ }^{1}$ Internal Medicine, Hospital Beatriz Ângelo, Loures, Portugal \\ ${ }^{2}$ Internal Medicine, Centro Hospitalar Lisboa Ocidental, Lisbon, Portugal
}

Doi: 10.12890/2019_001134 - European Journal of Case Reports in Internal Medicine - @ EFIM 2019

Received: $27 / 04 / 2019$

Accepted: 08/05/2019

Published: 03/06/2019

How to cite this article: Gameiro R, Gomes R, Jorge V, Picado B, Martos Gonçalves F, Lomelino Araújo J. A paraneoplastic syndrome to remember: a case of disseminated intravascular coagulation in lung cancer. EJCRIM 2019;6: doi:10.12890/2019_001134.

Conflicts of Interests: The Authors declare that there are no competing interest

This article is licensed under a Commons Attribution Non-Commercial 4.0 License

\section{ABSTRACT}

Disseminated intravascular coagulation (DIC) is an acquired syndrome characterized by the widespread activation of coagulation. It can present as an acute life-threatening emergency or as a chronic process. Mortality is highly dependent on the reversibility of the aetiology and degree of coagulation impairment, so treatment of the underlying cause is vital. The authors present the case of a 57-year-old man whose inaugural presentation of lung cancer was chronic DIC, characterized by three thrombotic events, followed by acute DIC, culminating in death. Metastatic lung cancer was diagnosed only after death.

\section{LEARNING POINTS}

- Disseminated intravascular coagulation (DIC) is an acquired syndrome characterized by systemic intravascular activation of coagulation, originating from and causing damage to the microvasculature, which can present as an acute or chronic condition, manifested by bleeding or thrombotic events.

- Unexplained thrombotic events should raise suspicion of chronic DIC.

- Treatment will fail, despite supportive measures, if the cause of the DIC is not identified.

\section{KEYWORDS}

Disseminated intravascular coagulation, lung cancer, intracerebral bleeding

\section{CASE DESCRIPTION}

A 57-year-old man with a previous history of arterial hypertension, previous smoking (40 pack-years), deep vein thrombosis (DVT) on rivaroxaban and recent hospitalization for Takotsubo cardiomyopathy, was admitted into the emergency department with acute dyspnoea, haemoptysis and syncope. On admission, he was polypnoeic, hypoxaemic and hypotensive. Arterial blood gas showed type 1 respiratory failure $\left(\mathrm{pO}_{2} 54 \mathrm{mmHg}\right.$ ) and hypocapnia $\left(\mathrm{pCO}_{2} 30 \mathrm{mmHg}\right.$ ). Blood work revealed increased D-dimers (25.54 mg/l, normal <0.50) and EKG had an S1Q3T3 pattern. Thoracic CT angiography confirmed a pulmonary embolism, revealing a peripheral pulmonary embolism of the lower left lobe, bilateral pleural effusion and bilateral lower lobe consolidation. During hospitalization, the patient initially experienced easy fatigability, dyspnoea and hypoxaemia requiring a high fraction of inspired oxygen. Given the recurrent venous thrombosis while on anticoagulation, additional investigations were performed: thrombophilia testing showed mild positive lupus anticoagulant, negative anticardiolipin and anti-beta2-glycoprotein antibodies, and negative serologic tests for human immunodeficiency virus, hepatitis B and C; abdominopelvic CT revealed mild to moderate pleural effusion, left lower lobe consolidation and bilateral augmented suprarenal glands. The patient was discharged after 10 days on levofloxacin and referred to an internal medicine appointment. Despite inconclusive studies, in light of high suspicion of a paraneoplastic syndrome caused by an occult malignancy, we opted for anticoagulation with tinzaparin. 
A month later, the patient was readmitted for a spontaneous dorsal haematoma. Physical examination identified decreased breath sounds on the left lower lung and a dorsal haematoma measuring $10 \times 5 \mathrm{~cm}$. Laboratory analysis showed thrombocytopenia (103,000/ $\mu$, normal 150-400,000), a prolonged prothrombin time (PT) (18.7 sec, normal 10.6-13.5) and an increased international normalized ratio (INR) (1.59). Hypoxemia was identified $\left(\mathrm{pO}_{2} 51 \mathrm{mmHg}\right.$ ) and on chest radiography there was evidence of right pulmonary hilar infiltrate. DIC was considered and anticoagulation was stopped. There was clinical degradation with ecchymosis on venipuncture sites and haemoptysis, as well as worsening thrombocytopenia (93,000/ $\mathrm{ll}$ ), aggravated coagulation times (PT $26.0 \mathrm{sec}$, INR 2.23), immeasurable D-dimers (>32.50 $\mathrm{mg} / \mathrm{l}$ ) and hypofibrogenemia (162 mg/dl, normal 180-350). Thoracic CT excluded new pulmonary embolisms and showed lung parenchyma alterations, so bronchofibroscopy was scheduled.

Given the unfavourable evolution, the patient was transferred to the intensive care unit. During the following hours, there was sudden loss of consciousness, preceded by nausea and vomiting, with altered consciousness (Glasgow coma score 7). Head CT showed a right frontal haematoma with rupture to the subarachnoid and subdural space, associated with cerebral compression with subfalcine and central herniation. Mannitol, fibrinogen, fresh frozen plasma and a platelet pool were administered. Neurosurgery consultation considered the patient unfit for surgical intervention due to his multiple comorbidities and previous anticoagulation. Despite supportive measures, there was continuing intracranial bleeding (Fig. 1) and the patient ultimately evolved into brain death. Given the high suspicion of an occult malignancy, he was considered unfit for transplantation. After authorization, an autopsy was performed which identified a $1.5 \mathrm{~cm}$ left lower lobe tumour with ipsilateral and contralateral nodal metastasis, as well as contralateral lung, suprarenal glands and splenic metastasis. There were also posterior heart wall ischaemic lesions, despite permeable coronary vessels, acute pancreatitis, haemorrhagic kidney lesions and haemorrhagic cystitis.

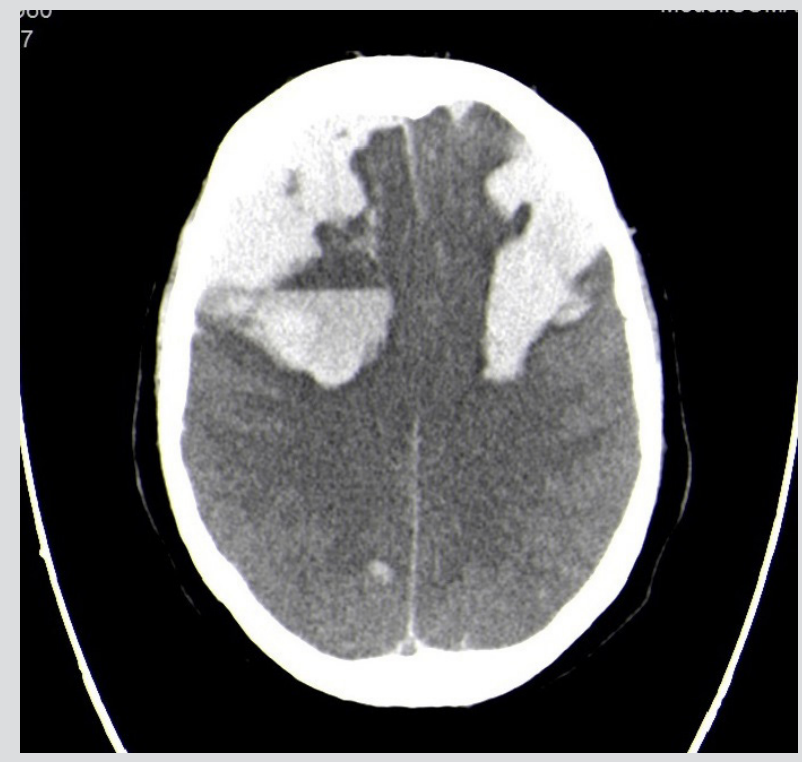

Figure 1. Axial section of head CT with extensive bilateral subarachnoid haemorrhage and ventricular flooding

\section{DISCUSSION}

Venous thromboembolism may be the inaugural presentation of neoplasia in an otherwise healthy person. Cancer patients have a four to seven times greater chance of developing thrombotic events, ranging from venous or arterial thrombosis to systemic syndromes, such as DIC. The main cancer-related risk factors are the primary tumour site, staging, therapy (mostly chemotherapy, hormonal and anti-angiogenic agents), utilization of erythropoiesis-stimulating agents, the presence of central venous catheters and blood transfusions ${ }^{[1]}$.

As proposed by the Scientific and Standardization Committee (SSC) of the International Society on Thrombosis and Haemostasis (ISTH), DIC is an acquired syndrome characterized by the intravascular activation of coagulation with loss of localization, originating from and causing damage to the microvasculature, which if sufficiently severe can cause organ dysfunction. It can present as an acute condition caused by a decompensated haemostatic system, designated as overt, acute or decompensated DIC, or as a chronic subclinical process, caused by a compensated haemostatic system, called non-overt, chronic or compensated DIC. It can present with various clinical features, ranging from abnormal coagulation tests to catastrophic thrombotic and bleeding manifestations. It seems to be underdiagnosed with an incidence of $10-15 \%$, emphasizing the need for clinical awareness of this condition ${ }^{[2]}$. DIC is emerging as a paraneoplastic syndrome and, as we know, 
trachea, bronchus and lung cancers were the sixth leading cause of death in 2016 according to World Health Organization statistics ${ }^{[3]}$. Regarding our clinical case, our main hypothesis had been a paraneoplastic syndrome linked to an occult neoplasia, given the recurrent thrombotic manifestations-the DVT, the myocardial infarction and the pulmonary embolism. When our patient presented with a spontaneous haematoma, generalized oozing from intravenous catheter sites and thrombocytopenia, our main hypothesis was acute DIC. All laboratory markers were present: trend reduction in thrombocytopenia, prolongation of coagulation times, hypofibrinogenemia and increased D-dimer. The trend reduction reflected worsening DIC $[2,4,5]$. We had not identified an obvious underlying condition, so we could only offer supportive measures. There was not sufficient time for a diagnosis given the catastrophic evolution. Ultimately, there were distinct signs of a worse outcome: central nervous system effects, worsening laboratory abnormalities and stage IV lung cancer.

Should we have suspected DIC all along? Could we have diagnosed cancer sooner? Would it have made a difference? We are aware of acute DIC with bleeding and thrombotic manifestations, but not of chronic DIC with laboratory-only alterations or localized thromboembolic events. Chronic DIC can progress to acute DIC when consumption of platelets and coagulation factors exceeds their production and the liver can no longer clear the fibrin degradation products ${ }^{[6]}$. In short, our clinical case can be interpreted as chronic DIC that culminated in acute DIC.

Even if we had previously diagnosed cancer, solid tumours do not respond as well to standard treatment as other DIC causes, despite equal outcome $^{[1,4]}$. Regarding mortality, it appears to be higher among lung cancer patients, especially in early stage and advanced malignancies, as in the case presented ${ }^{[1,4,7]}$. Moreover, it remains unclear whether the coagulopathy in itself carries a worse outcome or if it rather represents an epiphenomenon of an underlying disease with a worse prognosis. Risk factors for death include increasing age, severity of organ dysfunction and haemostatic abnormalities, the last two presented by our patient ${ }^{[2,4,8]}$.

This case is an example of a catastrophic paraneoplastic syndrome caused by lung cancer identified only after death. It reinforces the strong relationship between unexplained venous thromboembolism and cancer, as well as the need for a higher degree of suspicion for DIC.

\section{REFERENCES}

1. Sallah S, Wan JY, Nguyen NP, et al. Disseminated intravascular coagulation in solid tumours: clinical and pathologic study. Thromb Haemost 2001;86:828.

2. Levi M, Toh CH, Thachil J, et al. Guidelines for the diagnosis and management of disseminated intravascular coagulation. Br J Haematol 2009;145:24-33.

3. World Health Organization. Top 10 causes of death. 2016. https://www.who.int/gho/mortality_burden_disease/causes_death/top_10/en/ (accessed 3 April 2019).

4. Falanga A, Russo L, Milesi V, et al. Mechanisms and risk factors of thrombosis in cancer. Crit Rev Oncol Hematol 2017;118:79-83.

5. Wada H, Thachil J, Di Nisio M, et al. Guidance for diagnosis and treatment of disseminated intravascular coagulation from harmonization of the recommendations from three guidelines. J Thromb Haemost 2013;11:761-767.

6. Leung L. Clinical features, diagnosis, and treatment of disseminated intravascular coagulation in adults. UpToDate. https://www.uptodate.com/contents/clinical-featuresdiagnosis-and-treatment-of-disseminated-intravascular-coagulation-in-adults (accessed 3 April 2019).

7. Di Nisio M, Baudo F, Cosmi B, et al. Diagnosis and treatment of disseminated intravascular coagulation: guidelines of the Italian Society for Haemostasis and Thrombosis (SISET). Thromb Res 2012;129:e177-e184.

8. Nakano K, Sugiyama K, Satoh H, et al. Effect of thrombomodulin alfa on disseminated intravascular coagulation in patients with lung cancer. Intern Med 2017;56:1799-1806. 